\title{
Strong convergence of a new composite iterative method for equilibrium problems and fixed point problems in Hilbert spaces
}

\author{
Vahid Darvish and S. M. Vaezpour* \\ Department of Mathematics and Computer Science, \\ Amirkabir University of Technology, \\ Hafez Ave., P.O. Box 15875-4413, Tehran, Iran
}

September 15, 2018

\begin{abstract}
In this paper, first we introduce a new mapping for finding a common fixed point of an infinite family of nonexpansive mappings then we consider iterative method for finding a common element of the set of fixed points of an infinite family of nonexpansive mappings, the set of solutions of an equilibrium problem and the set of solutions of the variational inequality for $\alpha$-inverse-strongly monotone mapping in a Hilbert space. We show that under suitable conditions, the sequence converges strongly to a common element of the above three sets. Our results presented in this paper improve and extend other results.
\end{abstract}

Keywords: Variational inequality, Fixed point, Equilibrium problem, Nonexpansive mapping, $W_{n^{-}}$ mapping, $\alpha$-inverse-strongly monotone mapping.

2000 Mathematics Subject Classification: 47H10, 47H05, 47J05, 47J25

\section{Introduction and preliminaries}

Throughout this paper, we always assume that $H$ is a real Hilbert space with inner product, $\langle.,$.$\rangle and$ norm, $\|$.$\| , respectively and C$ is a nonempty closed convex subset of $H$. We use $\mathrm{F}(\mathrm{T})$ to denote the set of fixed point of $T$. Recall that a mapping $T: C \longrightarrow C$ is called nonexpansive if $\|T x-T y\| \leq\|x-y\|, \forall x, y \in$ $C$, and is a contraction, if there exists a constant $\alpha \in(0,1)$ such that $\|T x-T y\| \leq \alpha\|x-y\|, \forall x, y \in C$. Let $B: C \longrightarrow H$ be a mapping, the classical variational inequality problem is to find a $u \in C$ such that,

$$
\langle B u, v-u\rangle \geq 0, \forall v \in C .
$$

The set of solution of variational inequality (1.1) is denoted by VI(B,C).

An operator $A$ is said to be strongly positive if there exists a constant $\bar{\gamma}>0$ such that, $\langle A x, x\rangle \geq$ $\bar{\gamma}\|x\|^{2}, \forall x \in H$. It is easy to see that the following is true,

$$
u \in V I(B, C) \Longleftrightarrow u=P_{C}(u-\lambda B u), \lambda>0 .
$$

Let $F$ be a bifunction of $C \times C \longrightarrow \mathbb{R}$. The equilibrium problem for $F: C \times C \longrightarrow \mathbb{R}$ is to find $x \in C$ such that,

$$
F(x, y) \geq 0
$$

* Corresponding author.

E-mail addresses:

vahiddarvish@aut.ac.ir (V. Darvish),

vaez@aut.ac.ir (S. M. Vaezpour). 
for all $y \in C$, the set of solution of (1.2) is denoted by $\operatorname{EP}(\mathrm{F})$. It is well known that for every point $x \in H$, there exists a unique nearest point in $C$, denoted by $P_{C} x$, such that $\left\|x-P_{C} x\right\| \leq\|x-y\|, \forall y \in C$.

$P_{C}$ is called the metric projection of $H$ onto $C$ and is a nonexpansive mapping which satisfies

$$
\left\langle x-y, P_{C} x-P_{C} y\right\rangle \geq\left\|P_{C} x-P_{C} y\right\|^{2}, \forall x, y \in H .
$$

Moreover, $P_{C} x$ is characterized by the following properties, $P_{C} x \in C$ and

$$
\begin{gathered}
\left\langle x-P_{C} x, y-P_{C} y\right\rangle \leq 0, \\
\|x-y\|^{2} \geq\left\|x-P_{C} x\right\|^{2}+\left\|y-P_{C} x\right\|^{2},
\end{gathered}
$$

for all $x \in H, y \in C$.

For solving the equilibrium problem for a bifunctional $F: C \times C \longrightarrow \mathbb{R}$, let us assume that $F$ satisfies the following conditions:

(A1) $F(x, x)=0$, for all $x \in C$;

(A2) $F$ is monotone, i.e., $F(x, y)+F(y, x) \leq 0$, for all $x, y \in C$;

(A3) for each $x, y, z \in C, \lim _{t \rightarrow 0} F(t z+(1-t) x, y) \leq F(x, y)$;

(A4) for each $x \in C, y \longmapsto F(x, y)$ is convex and lower semicontinuous.

Recall that a mapping $B: C \longrightarrow H$ is said to be:

(i) monotone if $\langle B u-B v, u-v\rangle \geq 0, \forall u, v \in C$;

(ii) $L$-Lipschitz if there exists a constant $L>0$ such that $\|B u-B v\| \leq L\|u-v\|$, for all $u, v \in C$;

(iii) $\alpha$-inverse-strongly monotone, if there exists a positive real number $\alpha$ such that

$$
\langle B u-B v, u-v\rangle \geq \alpha\|B u-B v\|^{2}, \forall u, v \in C .
$$

We know that any $\alpha$-inverse-strongly monotone mapping $B$ is monotone and $\left(\frac{1}{\alpha}\right)$-Lipschitz continuous.

Numerous problems in physics, optimization and economics are to find a solution of (1.2). Let $A$ be strongly positive bounded linear operator on $H$, a typical problem is to minimize a quadratic function over the set of the fixed points of a nonexpansive mapping on a real Hilbert space H, i.e.,

$$
\min _{x \in F(T)} \frac{1}{2}\langle A x, x\rangle-\langle x, b\rangle
$$

where $b$ is a given point in $H$.

Takahashi $[8$ introduced an iterative method for finding a common element of equilibrium points of $F$ and the set of fixed points of a nonexpansive mapping in Hilbert spaces as follows:

$$
\begin{aligned}
& F\left(u_{n}, y\right)+\frac{1}{r_{n}}\left\langle y-u_{n}, u_{n}-x_{n}\right\rangle \geq 0, \forall y \in C, \\
& x_{n+1}=\alpha_{n} f\left(x_{n}\right)+\left(1-\alpha_{n}\right) T u_{n} .
\end{aligned}
$$

They proved under certain conditions $\left\{x_{n}\right\}$ and $\left\{u_{n}\right\}$ converges strongly to $z \in F(T) \bigcap E P(F)$, where $z \in P_{F(T)} \cap E P(F) f(z)$.

Moreover, Plubtieng and Pungpaeng 4 introduced the following iterative scheme:

$$
\begin{aligned}
& F\left(u_{n}, y\right)+\frac{1}{r_{n}}\left\langle y-u_{n}, u_{n}-x_{n}\right\rangle \geq 0, \forall y \in H, \\
& x_{n+1}=\alpha_{n} \gamma f\left(x_{n}\right)+\left(I-\alpha_{n} A\right) T u_{n}, \forall n \in \mathbb{N} .
\end{aligned}
$$


They proved under certain appropriate conditions on $\left\{\alpha_{n}\right\}$ and $\left\{r_{n}\right\}$ the sequence $\left\{x_{n}\right\}$ and $\left\{u_{n}\right\}$ generated by above scheme converges strongly to the unique solution of the variational inequality $\langle(A-$ $\gamma f) z, x-z\rangle \geq 0, \forall x \in F(T) \bigcap E P(F)$.

Zhao and He [11] introduced the following process:

$$
\begin{aligned}
& F\left(u_{n}, y\right)+\frac{1}{r_{n}}\left\langle y-u_{n}, u_{n}-x_{n}\right\rangle \geq 0, \forall y \in C, \\
& y_{n}=s_{n} P_{C}\left(u_{n}-\lambda_{n} A u_{n}\right)+\left(1-s_{n}\right) x_{n}, \\
& x_{n+1}=\alpha_{n} u+\beta_{n} x_{n}+\gamma_{n} W_{n}\left(P_{C}\left(y_{n}-\lambda_{n} A y_{n}\right)\right) .
\end{aligned}
$$

They proved under some conditions the sequences $\left\{x_{n}\right\}$ and $\left\{u_{n}\right\}$ converges strongly to $z \in \bigcap_{i=1}^{\infty} F\left(T_{i}\right) \bigcap V I(A, C) \bigcap E P(F)$, where $W_{n}$ is defined by Shimoji and Takahashi [6], as follows:

$$
\begin{aligned}
U_{n, n+1} & =I, \\
U_{n, n} & =\gamma_{n} T_{n} U_{n, n+1}+\left(1-\gamma_{n}\right) I, \\
U_{n, n-1} & =\gamma_{n-1} T_{n-1} U_{n, n}+\left(1-\gamma_{n-1}\right) I, \\
& \vdots \\
U_{n, k} & =\gamma_{k} T_{k} U_{n, k+1}+\left(1-\gamma_{k}\right) I, \\
U_{n, k-1} & =\gamma_{k-1} T_{k-1} U_{n, k}+\left(1-\gamma_{k-1}\right) I, \\
& \vdots \\
U_{n, 2} & =\gamma_{2} T_{2} U_{n, 3}+\left(1-\gamma_{2}\right) I, \\
W_{n} & =U_{n, 1}=\gamma_{1} T_{1} U_{n, 2}+\left(1-\gamma_{1}\right) I,
\end{aligned}
$$

where $\gamma_{1}, \gamma_{2}, \ldots$ are real numbers such that $0 \leq \gamma_{n} \leq 1, T_{1}, T_{2}, \ldots$ are an infinite family of mappings of $H$ into itself. Note that the nonexpansivity of each $T_{i}$ ensures the nonexpansivity of $W_{n}$.

Qin, Cho and Kang [5] introduced the following iterative scheme:

$$
\begin{aligned}
x_{1} & =x \in H, \text { chosen arbitrary, } \\
z_{n} & =\lambda_{n} x_{n}+\left(1-\lambda_{n}\right) W_{n} x_{n}, \\
y_{n} & =\beta_{n} \gamma f\left(z_{n}\right)+\left(I-\beta_{n} A\right) z_{n}, \\
x_{n+1} & =\alpha_{n} x_{n}+\left(1-\alpha_{n}\right) y_{n}, \quad \forall n \geq 1 .
\end{aligned}
$$

They proved that under certain assumptions on the sequences $\left\{\alpha_{n}\right\},\left\{\beta_{n}\right\}$ and $\left\{\lambda_{n}\right\},\left\{x_{n}\right\}$ converges strongly to a common fixed point of the infinite family of nonexpansive mappings.

Throughout this paper, inspired by Qin, Cho and Kang [5] and Zhao and He [1], we introduce a composite iterative method for infinite family of nonexpansive mappings as follows: 


$$
\begin{aligned}
& F\left(u_{n}, y\right)+\frac{1}{r_{n}}\left\langle y-u_{n}, u_{n}-x_{n}\right\rangle \geq 0, \forall y \in C, \\
& z_{n}=\lambda_{n} u_{n}+\left(1-\lambda_{n}\right) W_{n} u_{n} \\
& y_{n}=\beta_{n} \gamma f\left(z_{n}\right)+\left(I-\beta_{n} A\right) P_{C}\left(z_{n}-\gamma_{n} B z_{n}\right), \\
& x_{n+1}=\alpha_{n} x_{n}+\left(1-\alpha_{n}\right) y_{n}
\end{aligned}
$$

where $W_{n}$ is defined as (1.4) $f$ is a contraction on $H, A$ is strongly positive bounded linear self-adjoint operator, $B$ is $\alpha$-inverse-strongly monotone. We prove under certain appropriate assumptions on the sequences $\left\{\alpha_{n}\right\},\left\{\beta_{n}\right\},\left\{\lambda_{n}\right\}$ and $\left\{\gamma_{n}\right\}$, the sequence $\left\{x_{n}\right\}$ defined by (1.6), converges strongly to a common element of the set of fixed points of a finite family of nonexpansive mappings, the set of solutions of an equilibrium problem and the set of solutions of the variational inequality for $\alpha$-inverse-strongly monotone mapping.

We need the following lemmas for the proof of our main results.

Lemma 1.1. The following inequality holds in a Hilbert space $H$,

$$
\|x+y\|^{2} \leq\|x\|^{2}+2\langle y, x+y\rangle, \forall x, y \in H .
$$

Lemma 1.2. [10] Assume $\left\{\alpha_{n}\right\}$ is a sequence of nonnegative real numbers such that $\alpha_{n+1} \leq\left(1-\gamma_{n}\right) \alpha_{n}+$ $\delta_{n}, \quad n \geq 1$, where $\left\{\gamma_{n}\right\}$ is a sequence in $(0,1)$ and $\delta_{n}$ is a sequence in $\mathbb{R}$ such that:

1. $\sum_{n=1}^{\infty} \gamma_{n}=\infty$,

2. $\lim \sup _{n \rightarrow \infty}\left(\frac{\delta_{n}}{\gamma_{n}}\right) \leq 0$ or $\sum_{n=1}^{\infty}\left|\delta_{n}\right|<\infty$,

then $\lim _{n \rightarrow \infty} \alpha_{n}=0$.

Lemma 1.3. [3] Assume that $A$ is a strongly positive linear bounded self-adjoint operator on a Hilbert space $H$ with coefficient $\bar{\gamma}$ and $0<\rho \leq\|A\|^{-1}$, then $\|I-\rho A\| \leq 1-\rho \bar{\gamma}$.

Lemma 1.4. [7] Let $\left\{x_{n}\right\}$ and $\left\{y_{n}\right\}$ be bounded sequences in Banach space $X$ and let $\left\{\beta_{n}\right\}$ be a sequence in $[0,1]$ with $0<\liminf _{n \rightarrow \infty} \beta_{n} \leq \limsup _{n \rightarrow \infty} \beta_{n}<1$. Suppose that $x_{n+1}=\left(1-\beta_{n}\right) y_{n}+\beta_{n} x_{n}$ for all integers $n \geq 0$ and

$$
\limsup _{n \rightarrow \infty}\left(\left\|y_{n+1}-y_{n}\right\|-\left\|x_{n+1}-x_{n}\right\|\right) \leq 0 .
$$

Then, $\lim _{n \rightarrow \infty}\left\|y_{n}-x_{n}\right\|=0$.

Lemma 1.5. [1] Let $C$ be a nonempty closed convex subset of $H$ and let $F$ be a bifunction of $C \times C$ into $R$ satisfying (A1)-(A4). Let $r>0$ and $x \in H$. Then, there exists $z \in C$ such that,

$$
F(z, y)+\frac{1}{r}\langle y-z, z-x\rangle \geq 0, \forall y \in C .
$$

Lemma 1.6. [2] Assume that $F: C \times C \longrightarrow R$ satisfies (A1)-(A4). For $r>0$ and $x \in H$, define a mapping $T_{r}: H \longrightarrow C$ as follows:

$T_{r}(x)=\left\{z \in C: F(z, y)+\frac{1}{r}\langle y-z, z-x\rangle \geq 0, \forall y \in C\right\}$ for all $z \in H$. Then the following hold:

1. $T_{r}$ is single-valued;

2. $T_{r}$ is firmly nonexpansive, i.e., for any $x, y \in H,\left\|T_{r} x-T_{r} y\right\|^{2} \leq\left\langle T_{r} x-T_{r} y, x-y\right\rangle$; 
3. $F\left(T_{r}\right)=E P(F)$;

4. $\operatorname{EP}(F)$ is closed and convex.

Lemma 1.7. [6] Let $C$ be nonempty closed convex subset of a Hilbert space, let $T_{i}: C \longrightarrow C$ be an infinite family of nonexpansive mappings with $\bigcap_{i=1}^{\infty} F\left(T_{i}\right) \neq \emptyset$ and let $\gamma_{i}$ be a real sequence such that $0<\gamma_{i} \leq \gamma<1$ for all $i \geq 1$ then,

1. $W_{n}$ is nonexpansive and $F\left(W_{n}\right)=\bigcap_{i=1}^{n} F\left(T_{i}\right)$ for each $n \geq 1$,

2. For each $x \in C$ and for each positive integer $k$, the $\lim _{n \rightarrow \infty} U_{n, k}$ exists,

3. The mapping $W: C \longrightarrow C$ defined by :

$$
W x:=\lim _{n \rightarrow \infty} W_{n} x=\lim _{n \rightarrow \infty} U_{n, 1} x \quad x \in C,
$$

is a nonexpansive mapping satisfying $F(W)=\bigcap_{i=1}^{\infty} F\left(T_{i}\right)$ and is called the $W$-mapping generated by $T_{1}, T_{2}, \ldots$ and $\gamma_{1}, \gamma_{2}, \ldots$.

Lemma 1.8. [6] Let $C$ be a nonempty closed convex subset of a Hilbert space $H$, let $T_{i}: C \longrightarrow C$ be an infinite family of nonexpansive mappings with $\bigcap_{i=1}^{\infty} F\left(T_{i}\right) \neq \emptyset$ and let $\gamma_{i}$ be a real sequence such that $0<\gamma_{i} \leq \gamma<1$ for all $i \geq 1$, if $K$ is any bounded subset of $C$ then,

$$
\limsup _{n \rightarrow \infty}\left\|W x-W_{n} x\right\|=0, \quad x \in K .
$$

\section{Main Results}

In this section, we prove strong convergence theorem.

Theorem 2.1. Let $H$ be a real Hilbert space. Let $F$ be a bifunction from $C \times C \longrightarrow \mathbb{R}$ satisfying (A1)$\left(A_{4}\right)$ and let $B$ be an $\alpha$-inverse-strongly monotone mapping of $C$ into $H$, and let $\left\{T_{i}: C \longrightarrow C\right\}$ be a infinite family of nonexpansive mappings with $F:=\bigcap_{i=1}^{\infty} F\left(T_{i}\right) \cap V I(B, C) \bigcap E P(F) \neq \emptyset$. Suppose $A$ is a strongly positive linear bounded self-adjoint operator with the coefficient $\bar{\gamma} \geq 0$ and $0<\gamma \leq \frac{\bar{\gamma}}{\alpha}$. Let $\left\{\alpha_{n}\right\},\left\{\beta_{n}\right\},\left\{\lambda_{n}\right\}$ and $\left\{\delta_{n}\right\}$ be sequences in $[0,1]$ satisfying the following conditions:

(C1) $\sum_{n=0}^{\infty} \beta_{n}=\infty, \lim _{n \rightarrow \infty} \beta_{n}=0$;

(C2) $\sum_{i=1}^{\infty}\left|\lambda_{n}-\lambda_{n+1}\right|<\infty$;

(C3) $\sum_{i=1}^{\infty}\left|\alpha_{n}-\alpha_{n+1}\right|<\infty$;

(C4) $\exists \lambda \in[0,1] ; \lambda_{n}<\lambda, \forall n \geq 1$;

(C5) $\sum_{n=1}^{\infty}\left|\gamma_{n}-\gamma_{n+1}\right|<\infty, \gamma_{n} \in[a, b], a, b \in(0,2 \alpha)$;

(C6) $\liminf _{n \rightarrow \infty} r_{n}>0$ and $\sum_{n=1}^{\infty}\left|r_{n+1}-r_{n}\right|<\infty$.

Then the sequences $\left\{x_{n}\right\}$ defined by (1.6) converges strongly to $q \in F$, where $q=P_{F}(\gamma f+(I-A))(q)$ which solves the following variational inequality:

$$
\langle\gamma f(q)-A q, p-q\rangle \leq 0, \forall p \in F
$$

proof : For all $x, y \in C$ and $\gamma_{n} \in(0,2 \alpha)$, we note that 


$$
\begin{aligned}
\left\|\left(I-\gamma_{n} B\right) x-\left(I-\gamma_{n} B\right) y\right\|^{2} & =\left\|(x-y)-\gamma_{n}(B x-B y)\right\|^{2} \\
& =\|x-y\|^{2}-2 \gamma_{n}\langle x-y, B x-B y\rangle+\gamma_{n}^{2}\|B x-B y\|^{2} \\
& \leq\|x-y\|^{2}+\gamma_{n}\left(\gamma_{n}-2 \alpha\right)\|B x-B y\|^{2} \\
& \leq\|x-y\|^{2},
\end{aligned}
$$

which implies that $I-\gamma_{n} B$ is nonexpansive. Noticing that $A$ is a linear bounded self-adjoint operator so we have,

$$
\|A\|=\sup \{|\langle A x, x\rangle|: x \in H,\|x\|=1\} .
$$

Since $\beta_{n} \rightarrow 0$ as $n \rightarrow \infty$, by (C1) we may assume without loss of generality $\beta_{n}<\|A\|^{-1}, \forall n \in \mathbb{N}$.

$$
\left\langle\left(I-\beta_{n} A x\right), x\right\rangle=1-\beta_{n}\langle A x, x\rangle \geq 1-\beta_{n}\|A\| \geq 0,
$$

So $I-\beta_{n} A$ is positive.

It follows that,

$$
\begin{aligned}
\left\|I-\beta_{n} A\right\| & =\sup \left\{\left\langle\left(I-\beta_{n} A\right) x, x\right\rangle: x \in H,\|x\|=1\right\} \\
& =\sup \left\{1-\beta_{n}\langle A x, x\rangle: x \in H,\|x\|=1\right\} \\
& \leq 1-\beta_{n} \bar{\gamma} .
\end{aligned}
$$

Let $p \in \bigcap_{i=1}^{\infty} F\left(T_{i}\right) \cap V I(B, C) \cap E P(F)$ and $\left\{T_{r_{n}}\right\}$ be a sequence of mapping defined as in lemma (1.6), then $p=P_{C}\left(p-\gamma_{n} B p\right)=T_{r_{n}} p$. Put $t_{n}=P_{C}\left(z_{n}-\gamma_{n} B z_{n}\right)$, from (2.7) we have,

$$
\begin{aligned}
\left\|t_{n}-p\right\| & =\left\|P_{C}\left(z_{n}-\gamma_{n} B z_{n}\right)-P_{C}\left(p-\gamma_{n} B p\right)\right\| \\
& \leq\left\|z_{n}-\gamma_{n} B z_{n}-p+\gamma_{n} B p\right\| \\
& \leq\left\|z_{n}-p\right\| .
\end{aligned}
$$

Next we show that $\left\{x_{n}\right\}$ is bounded. Let $p \in F$, from the definition of $T_{r}$, we know that $u_{n}=T_{r_{n}} x_{n}$, it follows that $\left\|u_{n}-p\right\|=\left\|T_{r_{n}} x_{n}-T_{r_{n}} p\right\| \leq\left\|x_{n}-p\right\|$. Furthermore we have,

$$
\begin{aligned}
\left\|z_{n}-p\right\| & =\left\|\lambda_{n} u_{n}+\left(1-\lambda_{n}\right) W_{n} u_{n}-p\right\| \\
& =\left\|\lambda_{n}\left(u_{n}-p\right)+\left(1-\lambda_{n}\right)\left(W_{n} u_{n}-p\right)\right\| \\
& \leq \lambda_{n}\left\|u_{n}-p\right\|+\left(1-\lambda_{n}\right)\left\|W_{n} u_{n}-p\right\| \\
& =\left\|u_{n}-p\right\| \leq\left\|x_{n}-p\right\|,
\end{aligned}
$$

and hence, 


$$
\begin{aligned}
\left\|y_{n}-p\right\| & =\left\|\beta_{n} \gamma f\left(z_{n}\right)+\left(\left(I-\beta_{n} A\right)\right) P_{C}\left(z_{n}-\gamma_{n} B z_{n}\right)-p\right\| \\
& =\left\|\beta_{n}\left(\gamma f\left(z_{n}\right)-A p\right)+\left(I-\beta_{n} A\right)\left(P_{C}\left(z_{n}-\gamma_{n} B z_{n}\right)-p\right)\right\| \\
& \leq \beta_{n}\left\|\gamma f\left(z_{n}\right)-A p\right\|+\left(1-\beta_{n} \bar{\gamma}\right)\left\|z_{n}-p\right\| \\
& \leq \beta_{n} \gamma\left\|f\left(z_{n}\right)-f(p)\right\|+\beta_{n}\|\gamma f(p)-A p\|+\left(1-\beta_{n} \bar{\gamma}\right)\left\|z_{n}-p\right\| \\
& \leq \beta_{n} \gamma \alpha\left\|z_{n}-p\right\|+\beta_{n}\|\gamma f(p)-A p\|+\left(1-\beta_{n} \bar{\gamma}\right)\left\|z_{n}-p\right\| \\
& \leq \beta_{n} \gamma \alpha\left\|x_{n}-p\right\|+\beta_{n}\|\gamma f(p)-A p\|+\left(1-\beta_{n} \bar{\gamma}\right)\left\|x_{n}-p\right\| \\
& =\left(1-\beta_{n}(\bar{\gamma}-\gamma \alpha)\right)\left\|x_{n}-p\right\|+\beta_{n}\|\gamma f(p)-A p\| .
\end{aligned}
$$

It follows that,

$$
\begin{aligned}
\left\|x_{n+1}-p\right\| & =\left\|\alpha_{n} x_{n}+\left(1-\alpha_{n}\right) y_{n}-p\right\| \\
& \leq \alpha_{n}\left\|x_{n}-p\right\|+\left(1-\alpha_{n}\right)\left\|y_{n}-p\right\| \\
& \leq \alpha_{n}\left\|x_{n}-p\right\|+\left(1-\alpha_{n}\right)\left[\left(1-\beta_{n}(\bar{\gamma}-\gamma \alpha)\right)\left\|x_{n}-p\right\|+\beta_{n}\|\gamma f(p)-A p\|\right] \\
& =\left[1-\beta_{n}(\bar{\gamma}-\gamma \alpha)\left(1-\alpha_{n}\right)\right]\left\|x_{n}-p\right\|+\beta_{n}(\bar{\gamma}-\gamma \alpha)\left(1-\alpha_{n}\right) \frac{\|\gamma f(p)-A p\|}{\bar{\gamma}-\gamma \alpha}
\end{aligned}
$$

by simple induction we have,

$$
\left\|x_{n}-p\right\| \leq \max \left\{\left\|x_{1}-p\right\|, \frac{\|A p-\gamma f(p)\|}{\bar{\gamma}-\gamma \alpha}\right\} .
$$

Which gives that the sequence $\left\{x_{n}\right\}$ is bounded, so $\left\{y_{n}\right\}$ and $\left\{z_{n}\right\}$.

Next, we claim that $\lim _{n \rightarrow \infty}\left\|x_{n+1}-x_{n}\right\|=0$.

First we have $u_{n}=T_{r_{n}} x_{n}, u_{n-1}=T_{r_{n}} x_{n-1}$, so

$$
F\left(u_{n-1}, y\right)+\frac{1}{r_{n-1}}\left\langle y-u_{n-1}, u_{n-1}-x_{n-1}\right\rangle \geq 0, \forall y \in C,
$$

and

$$
F\left(u_{n}, y\right)+\frac{1}{r_{n}}\left\langle y-u_{n}, u_{n}-x_{n}\right\rangle \geq 0, \forall y \in C,
$$

putting $y=u_{n}$ in (2.8) and $y=u_{n-1}$ in (2.9), we have,

$$
F\left(u_{n-1}, u_{n}\right)+\frac{1}{r_{n-1}}\left\langle u_{n}-u_{n-1}, u_{n-1}-x_{n-1}\right\rangle \geq 0,
$$

and

$$
F\left(u_{n}, u_{n-1}\right)+\frac{1}{r_{n}}\left\langle u_{n-1}-u_{n}, u_{n}-x_{n}\right\rangle \geq 0 .
$$

Hence,

$$
\left\langle u_{n}-u_{n-1}, \frac{u_{n-1}-x_{n-1}}{r_{n-1}}-\frac{u_{n}-x_{n}}{r_{n}}\right\rangle \geq 0,
$$

therefore we have,

$$
\left\langle u_{n}-u_{n-1}, u_{n-1}-u_{n}+u_{n}-x_{n-1}-\frac{r_{n-1}}{r_{n}}\left(u_{n}-x_{n}\right)\right\rangle \geq 0 .
$$


We can assume that there is a real number $b$ such that $r_{n}>b>0$, for all $n \in \mathbb{N}$. Then we have,

$$
\begin{aligned}
\left\|u_{n}-u_{n-1}\right\|^{2} & \leq\left\langle u_{n}-u_{n-1}, x_{n}-x_{n-1}+\left(1-\frac{r_{n-1}}{r_{n}}\right)\left(u_{n}-x_{n}\right)\right\rangle \\
& \leq\left\|u_{n}-u_{n-1}\right\|\left[\left\|x_{n}-x_{n-1}\right\|+\left|1-\frac{r_{n-1}}{r_{n}}\right|\left\|u_{n}-x_{n}\right\|\right] .
\end{aligned}
$$

So,

$$
\begin{aligned}
\left\|u_{n}-u_{n-1}\right\| & \leq\left\|x_{n}-x_{n-1}\right\|+\frac{1}{r_{n}}\left|r_{n}-r_{n-1}\right|\left\|u_{n}-x_{n}\right\| \\
& \leq\left\|x_{n}-x_{n-1}\right\|+\frac{1}{b}\left|r_{n}-r_{n-1}\right| L
\end{aligned}
$$

where $L=\sup \left\{\left\|u_{n}-x_{n}\right\|: n \in \mathbb{N}\right\}$.

On the other hand,

$$
\begin{aligned}
\left\|z_{n}-z_{n-1}\right\|= & \left\|\lambda_{n} u_{n}+\left(1-\lambda_{n}\right) W_{n} u_{n}-\lambda_{n-1} u_{n-1}-\left(1-\lambda_{n-1}\right) W_{n-1} u_{n-1}\right\| \\
\leq & \lambda_{n}\left\|u_{n}-u_{n-1}\right\|+\left|\lambda_{n}-\lambda_{n-1}\right|\left\|u_{n-1}-W_{n-1} u_{n-1}\right\| \\
& +\left(1-\lambda_{n}\right)\left\|W_{n} u_{n}-W_{n} u_{n-1}\right\|+\left(1-\lambda_{n}\right)\left\|W_{n} u_{n-1}-W_{n-1} u_{n-1}\right\| \\
\leq & \left\|u_{n}-u_{n-1}\right\|+\left|\lambda_{n}-\lambda_{n-1}\right|\left\|u_{n-1}-W_{n-1} u_{n-1}\right\|+\left(1-\lambda_{n}\right)\left\|W_{n} u_{n-1}-W_{n-1} u_{n-1}\right\| .
\end{aligned}
$$

Also by nonexpansivity of $T_{i}$ and the definition of $W_{n}$ we have,

$$
\begin{aligned}
\left\|W_{n} u_{n-1}-W_{n-1} u_{n-1}\right\| & =\left\|\mu_{1} T_{1} U_{n, 2} u_{n-1}-\left(1-\mu_{1}\right) u_{n-1}-\mu_{1} T_{1} U_{n-1,2} u_{n-1}-\left(1-\mu_{1}\right) u_{n-1}\right\| \\
& \leq \mu_{1}\left\|U_{n, 2} u_{n-1}-U_{n-1,2} u_{n-1}\right\| \\
& =\mu_{1}\left\|\mu_{2} T_{2} U_{n, 3} u_{n-1}-\left(1-\mu_{2}\right) u_{n-1}-\mu_{2} T_{2} U_{n-1,3} u_{n-1}-\left(1-\mu_{2}\right) u_{n-1}\right\| \\
& \leq \mu_{1} \mu_{2}\left\|U_{n, 3} u_{n-1}-U_{n-1,3} u_{n-1}\right\| \\
& \vdots \\
& \leq\left(\prod_{i=1}^{n-1} \mu_{i}\right)\left\|U_{n, n} u_{n-1}-U_{n-1, n} u_{n-1}\right\| \\
& \leq M_{1}\left(\prod_{i=1}^{n-1} \mu_{i}\right),
\end{aligned}
$$

where $M_{1} \geq 0$ is a constant such that $\left\|U_{n, n} u_{n-1}-U_{n-1, n} u_{n-1}\right\| \leq M_{1}$.

Now we have,

$\left\|z_{n}-z_{n-1}\right\|=\left\|u_{n}-u_{n-1}\right\|+\left|\lambda_{n}-\lambda_{n-1}\right|\left\|u_{n-1}-W_{n-1} u_{n-1}\right\|+\left(1-\lambda_{n}\right) M_{1}$.

Also,

$$
\begin{aligned}
\left\|y_{n}-y_{n-1}\right\| & =\left\|\beta_{n} \gamma f\left(z_{n}\right)+\left(I-\beta_{n} A\right) t_{n}-\beta_{n-1} \gamma f\left(z_{n-1}\right)-\left(I-\beta_{n-1} A\right) t_{n-1}\right\| \\
& \leq \beta_{n} \gamma \alpha\left\|z_{n}-z_{n-1}\right\|+\gamma\left|\beta_{n}-\beta_{n-1}\right|\left\|f\left(z_{n-1}\right)\right\|+\left(1-\beta_{n} \bar{\gamma}\right)\left\|t_{n}-t_{n-1}\right\|+\left|\beta_{n-1}-\beta_{n}\right|\left\|A t_{n-1}\right\|,
\end{aligned}
$$


where,

$$
\begin{aligned}
\left\|t_{n}-t_{n-1}\right\| & =\left\|P_{C}\left(z_{n}-\gamma_{n} B z_{n}\right)-P_{C}\left(z_{n-1}-\gamma_{n-1} B z_{n-1}\right)\right\| \\
& \leq\left\|\left(z_{n}-z_{n-1}\right)-\left(\gamma_{n} B z_{n}-\gamma_{n-1} B z_{n-1}\right)\right\| \\
& \leq\left\|z_{n}-z_{n-1}\right\|+\frac{\gamma_{n}}{\alpha}\left\|z_{n}-z_{n-1}\right\|+\left|\gamma_{n}-\gamma_{n-1}\right|\left\|B z_{n-1}\right\| .
\end{aligned}
$$

So,

$$
\begin{aligned}
\left\|y_{n}-y_{n-1}\right\| \leq & \beta_{n} \gamma \alpha\left\|z_{n}-z_{n-1}\right\|+\gamma\left|\beta_{n}-\beta_{n-1}\right|\left\|f\left(z_{n-1}\right)\right\|+\left|\beta_{n-1}-\beta_{n}\right|\left\|A t_{n-1}\right\| \\
+ & \left(1-\beta_{n} \bar{\gamma}\right)\left(\left(\frac{\alpha+\gamma_{n}}{\alpha}\right)\left\|z_{n}-z_{n-1}\right\|+\left|\gamma_{n}-\gamma_{n-1}\right|\left\|B z_{n-1}\right\|\right) \\
\leq & \left(\beta_{n} \gamma \alpha+\left(1-\beta_{n} \bar{\gamma}\right)\left(\frac{\alpha+\gamma_{n}}{\alpha}\right)\right)\left\|z_{n}-z_{n-1}\right\| \\
& +\left(\left|\gamma_{n}-\gamma_{n-1}\right|\left(1-\beta_{n} \bar{\gamma}\right)+\left|\beta_{n}-\beta_{n-1}\right|\right) M_{2},
\end{aligned}
$$

where, $M_{2}=\sup \left\{\gamma \| f\left(z_{n-1}\|+\| B z_{n-1}\|+\| A t_{n-1} \|\right\}\right.$

$$
\begin{aligned}
\leq & \left(1-\beta_{n}(\bar{\gamma}-\gamma \alpha)+\frac{\gamma_{n}}{\alpha}\right)\left[\left\|u_{n}-u_{n-1}\right\|\right. \\
& \left.+\left|\lambda_{n}-\lambda_{n-1}\right|\left\|u_{n-1}-W_{n-1} u_{n-1}\right\|+\left(1-\lambda_{n}\right) M_{1} \prod_{i=1}^{n-1} \mu_{i}\right] \\
& +p_{n} M_{2},
\end{aligned}
$$

where, $p_{n}=\left(\left|\gamma_{n}-\gamma_{n-1}\right|\left(1-\beta_{n} \bar{\gamma}\right)+\left|\beta_{n}-\beta_{n-1}\right|\right)$.

$$
\begin{aligned}
\leq & \left(1-\beta_{n}(\bar{\gamma} \alpha-\gamma \alpha)+\frac{\gamma_{n}}{\alpha}\right)\left[\left\|x_{n}-x_{n-1}\right\|+\frac{1}{b}\left|r_{n}-r_{n-1}\right| L\right] \\
& +\left(1+\frac{\gamma_{n}}{\alpha}\right)\left[\left|\lambda_{n}-\lambda_{n-1}\right|\left\|u_{n-1}-W_{n-1} u_{n-1}\right\|\right. \\
& \left.+\left(1-\lambda_{n}\right) M_{1} \prod_{i=1}^{n-1} \mu_{i}\right]+p_{n} M_{2} .
\end{aligned}
$$

We note that,

$$
\begin{aligned}
\left\|x_{n+1}-x_{n}\right\|= & \left\|\alpha_{n} x_{n}+\left(1-\alpha_{n}\right) y_{n}-\alpha_{n-1} x_{n-1}-\left(1-\alpha_{n-1}\right) y_{n-1}\right\| \\
= & \| \alpha_{n} x_{n}-\alpha_{n} x_{n-1}+\alpha_{n} x_{n-1}-\alpha_{n-1} x_{n-1}+\left(1-\alpha_{n}\right) y_{n}-\left(1-\alpha_{n}\right) y_{n-1} \\
& +\left(1-\alpha_{n}\right) y_{n-1}-\left(1-\alpha_{n-1}\right) y_{n-1} \| \\
\leq & \alpha_{n}\left\|x_{n}-x_{n-1}\right\|+\left|\alpha_{n}-\alpha_{n-1}\right|\left\|x_{n-1}\right\|+\left(1-\alpha_{n}\right)\left\|y_{n}-y_{n-1}\right\|+\left|\alpha_{n-1}-\alpha_{n}\right|\left\|y_{n-1}\right\| .
\end{aligned}
$$

If we put $K=\sup \left\{\left\|x_{n-1}\right\|+\left\|y_{n-1}\right\|\right\}$.

So, 
$\left\|x_{n+1}-x_{n}\right\| \leq \alpha_{n}\left\|x_{n}-x_{n-1}\right\|+2 K\left|\alpha_{n}-\alpha_{n-1}\right|+\left(1-\alpha_{n}\right)\left\|y_{n}-y_{n-1}\right\|$. By substitute $\left\|y_{n}-y_{n-1}\right\|$ in above inequality we have,

$$
\begin{aligned}
\left\|x_{n+1}-x_{n}\right\| \leq & \alpha_{n}\left\|x_{n}-x_{n-1}\right\|+\left(1-\alpha_{n}\right)\left(1-\beta_{n}(\bar{\gamma}-\gamma \alpha)+\frac{\gamma_{n}}{\alpha}\right)\left[\left\|x_{n}-x_{n-1}\right\|\right. \\
& \left.+\frac{1}{b}\left|r_{n}-r_{n-1}\right| L\right] \\
& +\left(1-\alpha_{n}\right)\left(1+\frac{\gamma_{n}}{\alpha}\right)\left[\left\|u_{n-1}-W_{n-1} u_{n-1}\right\|\left|\lambda_{n}-\lambda_{n-1}\right|\right. \\
& \left.+\left(1-\lambda_{n}\right) M_{1}\right]+\left(1-\alpha_{n}\right) p_{n} M_{2}+2 K\left|\alpha_{n}-\alpha_{n-1}\right| \\
= & \left(3-\beta_{n}(\bar{\gamma}-\gamma \alpha)\right)\left\|x_{n}-x_{n-1}\right\|+s_{n}
\end{aligned}
$$

where,

$$
\begin{aligned}
s_{n}= & \left\{\left(1-\alpha_{n}\right)\left(1-\beta_{n}(\bar{\gamma}-\gamma \alpha)+\frac{\gamma_{n}}{\alpha}\right)\left(\frac{1}{b}\left|r_{n}-r_{n-1}\right| L\right)\right. \\
& +\left(1-\alpha_{n}\right)\left(1+\frac{\gamma_{n}}{\alpha}\right)\left[|| u_{n-1}-W_{n-1} u_{n-1}||\left|\lambda_{n}-\lambda_{n-1}\right|+\left(1-\lambda_{n}\right) M_{1} \prod_{i=1}^{n-1} \mu_{i}\right] \\
& \left.+\left(1-\alpha_{n}\right) p_{n} M_{2}+2 K\left|\alpha_{n}-\alpha_{n-1}\right|\right\} .
\end{aligned}
$$

So,

$\left\|x_{n+1}-x_{n}\right\| \leq\left(1-\beta_{n}\left(\frac{\bar{\gamma}-\gamma \alpha}{3}\right)\right)\left\|x_{n}-x_{n-1}\right\|+s_{n}$, and by lemma (1.2), we have

$$
\left\|x_{n}-x_{n-1}\right\| \rightarrow 0
$$

Now we show that $\lim _{n \rightarrow \infty}\left\|y_{n}-u_{n}\right\|=0$. We have,

$$
\begin{aligned}
\left\|y_{n+1}-y_{n}\right\|= & \|\left(I-\beta_{n+1} A\right)\left(t_{n+1}-t_{n}\right)-\left(\beta_{n+1}-\beta_{n}\right) A t_{n} \\
& +\gamma\left[\beta_{n+1}\left(f\left(z_{n+1}\right)-f\left(z_{n}\right)\right)+f\left(z_{n}\right)\left(\beta_{n+1}-\beta_{n}\right)\right] \| \\
\leq & \left(1-\beta_{n+1} \bar{\gamma}\right)\left\|t_{n+1}-t_{n}\right\|+\left|\beta_{n+1}-\beta_{n}\right|\left\|A t_{n}\right\| \\
& +\gamma \beta_{n+1} \alpha\left\|z_{n+1}-z_{n}\right\|+\left|\gamma_{n+1}-\gamma_{n}\right|\left\|B z_{n}\right\| \\
& +\gamma\left|\beta_{n+1}-\beta_{n}\right|\left\|f\left(z_{n}\right)\right\| \\
\leq & \left(1-\beta_{n+1}(\bar{\gamma}-\gamma \alpha)\right)\left\|z_{n+1}-z_{n}\right\|+\left|\gamma_{n+1}-\gamma_{n}\right|\left\|B z_{n}\right\|+\left|\beta_{n+1}-\beta_{n}\right| M_{3} \\
\leq & \left\|z_{n+1}-z_{n}\right\|+\left|\gamma_{n+1}-\gamma_{n}\right|\left\|B z_{n}\right\|+\left|\beta_{n+1}-\beta_{n}\right| M_{3},
\end{aligned}
$$

where $M_{3}=\sup \left\{\left\|A t_{n}\right\|+\gamma\left\|f\left(z_{n}\right)\right\|\right\}$.

So,

$$
\begin{aligned}
\left\|y_{n+1}-y_{n}\right\| \leq & \left\|u_{n+1}-u_{n}\right\|+\left\|u_{n}-W_{n} u_{n}\right\|\left|\lambda_{n+1}-\lambda_{n}\right| \\
& +\left(1-\lambda_{n+1}\right) M_{1} \prod_{i=1}^{n-1} \mu_{i}+\left|\gamma_{n+1}-\gamma_{n}\right||| B z_{n} \|+\left|\beta_{n+1}-\beta_{n}\right| M_{3}
\end{aligned}
$$


which implies,

$$
\begin{aligned}
\left\|y_{n+1}-y_{n}\right\|-\left\|u_{n+1}-u_{n}\right\| \leq & \left\|u_{n}-W_{n} u_{n}\right\|\left|\lambda_{n+1}-\lambda_{n}\right|+\left(1-\lambda_{n+1}\right) M_{1} \prod_{i=1}^{n-1} \mu_{i} \\
& +\left|\beta_{n+1}-\beta_{n}\right| M_{3}+\left|\gamma_{n+1}-\gamma_{n}\right|\left\|B z_{n}\right\| .
\end{aligned}
$$

By conditions (C1), (C2) and (C5) we have, $\lim \sup \left\{\left\|y_{n+1}-y_{n}\right\|-\left\|u_{n+1}-u_{n}\right\|\right\} \leq 0$ and by lemma (1.4), we have $\lim _{\sup _{n \rightarrow \infty}}\left\|y_{n}-u_{n}\right\|=0$.

Also we show that $\lim _{n \rightarrow \infty}\left\|x_{n}-u_{n}\right\|=0$. We have,

$$
\begin{aligned}
\left\|x_{n+1}-p\right\|^{2} & =\left\|\alpha_{n} x_{n}+\left(1-\alpha_{n}\right) y_{n}-p\right\|^{2} \\
& \leq \alpha_{n}\left\|x_{n}-p\right\|^{2}+\left(1-\alpha_{n}\right)\left\|y_{n}-p\right\|^{2} \\
& \leq \alpha_{n}\left\|x_{n}-p\right\|^{2}+\left(1-\alpha_{n}\right)\left(\left\|y_{n}-u_{n}\right\|+\left\|u_{n}-p\right\|\right)^{2} \\
& =\alpha_{n}\left\|x_{n}-p\right\|^{2}+\left(1-\alpha_{n}\right)\left\|y_{n}-u_{n}\right\|^{2}+2\left(1-\alpha_{n}\right)\left\|y_{n}-u_{n}\right\|\left\|u_{n}-p\right\|+\left(1-\alpha_{n}\right)\left\|u_{n}-p\right\|^{2} .
\end{aligned}
$$

Also,

$$
\begin{aligned}
\left\|u_{n}-p\right\|^{2} & =\left\|T_{r_{n}} x_{n}-T_{r_{n}} p\right\|^{2} \leq\left\langle T_{r_{n}} x_{n}-T_{r_{n}} p, x_{n}-p\right\rangle \\
& =\left\langle u_{n}-p, x_{n}-p\right\rangle=\frac{1}{2}\left(\left\|u_{n}-p\right\|^{2}+\left\|x_{n}-p\right\|^{2}-\left\|x_{n}-u_{n}\right\|^{2}\right) .
\end{aligned}
$$

Now by (2.12) we have,

$$
\begin{aligned}
\left\|x_{n+1}-p\right\|^{2} & \leq \alpha_{n}\left\|x_{n}-p\right\|^{2}+\left(1-\alpha_{n}\right)\left(\left\|x_{n}-p\right\|^{2}-\left\|x_{n}-u_{n}\right\|^{2}\right)+d_{n} \\
& =\left\|x_{n}-p\right\|^{2}-\left(1-\alpha_{n}\right)\left\|x_{n}-u_{n}\right\|^{2}+d_{n},
\end{aligned}
$$

where,

$$
d_{n}=\left(1-\alpha_{n}\right)\left\|y_{n}-u_{n}\right\|^{2}+2\left(1-\alpha_{n}\right)\left\|y_{n}-u_{n}\right\|\left\|u_{n}-p\right\| .
$$

Therefore,

$$
\begin{aligned}
\left(1-\alpha_{n}\right)\left\|x_{n}-u_{n}\right\|^{2} & \leq\left\|x_{n}-p\right\|-\left\|x_{n+1}-p\right\|^{2}+d_{n} \\
& \leq\left\|x_{n}-x_{n+1}\right\|\left(\left\|x_{n}-p\right\|+\left\|x_{n+1}-p\right\|\right)+d_{n},
\end{aligned}
$$

by using (2.10), we have, $\lim _{n \rightarrow \infty}\left\|x_{n}-u_{n}\right\|=0$.

Now we can say that, $\left\|y_{n}-x_{n}\right\| \leq\left\|y_{n}-u_{n}\right\|+\left\|u_{n}-x_{n}\right\|$,

so,

$$
\lim _{n \rightarrow \infty}\left\|y_{n}-x_{n}\right\|=0
$$


Next we will show that, $\lim \left\|W t_{n}-t_{n}\right\| \rightarrow 0$.

First we prove that, $\lim _{n \rightarrow \infty}\left\|B z_{n}-B p\right\|=0$.

$$
\begin{aligned}
\left\|y_{n}-p\right\|^{2}= & \left\|\beta_{n} \gamma f\left(z_{n}\right)+\left(I-\beta_{n} A\right) t_{n}-p\right\|^{2} \\
= & \left\|\beta_{n}\left(\gamma f\left(z_{n}\right)-A p\right)+\left(I-\beta_{n} A\right)\left(t_{n}-p\right)\right\|^{2} \\
= & \left(1-\beta_{n} \bar{\gamma}\right)^{2}\left\|t_{n}-p\right\|^{2}+\beta_{n}^{2}\left\|\gamma f\left(z_{n}\right)-A p\right\|^{2} \\
& +2\left\langle\beta_{n}\left(\gamma f\left(z_{n}\right)-A p\right),\left(I-\beta_{n} A\right)\left(t_{n}-p\right)\right\rangle \\
\leq & \left(1-\beta_{n} \bar{\gamma}\right)^{2}\left\|z_{n}-\gamma_{n} B z_{n}-p+\gamma_{n} B p\right\|^{2}+\beta_{n}^{2}\left\|\gamma f\left(z_{n}\right)-A p\right\|^{2} \\
& +2\left\langle\beta_{n}\left(\gamma f\left(z_{n}\right)-A p\right),\left(I-\beta_{n} A\right)\left(t_{n}-p\right)\right\rangle \\
= & \left(1-\beta_{n} \bar{\gamma}\right)^{2}\left[\left\|z_{n}-p\right\|^{2}+\gamma_{n}\left(\gamma_{n}-2 \alpha\right)\left\|B z_{n}-B p\right\|^{2}\right]+c_{n},
\end{aligned}
$$

where, $c_{n}=\beta_{n}^{2}\left\|\gamma f\left(z_{n}\right)-A p\right\|^{2}+2\left\langle\beta_{n}\left(\gamma f\left(z_{n}\right)-A p\right),\left(I-\beta_{n} A\right)\left(t_{n}-p\right)\right\rangle$.

So,

$$
\begin{aligned}
\left(1-\beta_{n} \bar{\gamma}\right)^{2} b(b-2 \alpha)\left\|B z_{n}-B p\right\|^{2} \leq & \left(1-\beta_{n} \bar{\gamma}\right)\left\|u_{n}-p\right\|^{2}-\left\|y_{n}-p\right\|+c_{n} \\
\leq & \left.1-\beta_{n} \bar{\gamma}\right)^{2}\left(\left\|u_{n}-y_{n}\right\|+\left\|y_{n}-p\right\|\right)^{2}-\left\|y_{n}-p\right\|^{2}+c_{n} \\
\leq & \left(1-\beta_{n} \bar{\gamma}\right)^{2}\left[\left\|u_{n}-y_{n}\right\|^{2}+2\left\|y_{n}-u_{n}\right\|\left\|y_{n}-p\right\|+\left\|y_{n}-p\right\|^{2}\right] \\
& -\left\|y_{n}-p\right\|^{2}+c_{n},
\end{aligned}
$$

hence, $\lim _{n \rightarrow \infty}\left\|B z_{n}-B p\right\|=0$.

On the other hand,

$$
\begin{aligned}
\left\|t_{n}-p\right\|^{2}= & \left\|P_{C}\left(z_{n}-\gamma B z_{n}\right)-P_{C}\left(p-\gamma_{n} B p\right)\right\|^{2} \\
\leq & \left\langle\left(z_{n}-\gamma_{n} B z_{n}\right)-\left(p-\gamma_{n} B p\right), t_{n}-p\right\rangle \\
= & \frac{1}{2}\left\{\left\|\left(z_{n}-\gamma_{n} B z_{n}\right)-\left(p-\gamma_{n} B p\right)\right\|^{2}+\left\|t_{n}-p\right\|^{2}\right. \\
& \left.-\left\|\left(z_{n}-\gamma_{n} B z_{n}\right)-\left(p-\gamma_{n} B p\right)-\left(t_{n}-p\right)\right\|^{2}\right\} \\
\leq & \frac{1}{2}\left\{\left\|z_{n}-p\right\|^{2}+\left\|t_{n}-p\right\|^{2}-\left\|\left(z_{n}-t_{n}\right)-\gamma_{n}\left(B z_{n}-B p\right)\right\|^{2}\right\} \\
= & \frac{1}{2}\left\{\left\|z_{n}-p\right\|^{2}+\left\|t_{n}-p\right\|^{2}-\left\|z_{n}-t_{n}\right\|^{2}\right. \\
& \left.+2 \gamma_{n}\left\langle z_{n}-t_{n}, B z_{n}-B p\right\rangle-\gamma_{n}^{2}\left\|B z_{n}-B p\right\|^{2}\right\},
\end{aligned}
$$

which implies,

$\left\|t_{n}-p\right\|^{2} \leq\left\|z_{n}-p\right\|^{2}-\left\|z_{n}-t_{n}\right\|^{2}+2 \gamma_{n}\left\langle z_{n}-t_{n}, B z_{n}-B p\right\rangle-\gamma_{n}^{2}\left\|B z_{n}-B p\right\|^{2}$.

Now by above inequality we want to show that, $\left\|z_{n}-t_{n}\right\| \rightarrow 0$.

$$
\begin{aligned}
\left\|y_{n}-p\right\|^{2}= & \left(1-\beta_{n} \bar{\gamma}\right)^{2}\left\|t_{n}-p\right\|^{2}+c_{n} \\
\leq & \left(1-\beta_{n} \bar{\gamma}\right)^{2}\left(\left\|z_{n}-p\right\|^{2}-\left\|z_{n}-t_{n}\right\|^{2}+2 \gamma_{n}\left\langle z_{n}-t_{n}, B z_{n}-B p\right\rangle\right. \\
& \left.-\gamma_{n}^{2}\left\|B z_{n}-B p\right\|^{2}\right)+c_{n} .
\end{aligned}
$$

Therefore, 


$$
\begin{aligned}
\left(1-\beta_{n} \bar{\gamma}\right)^{2}\left\|z_{n}-t_{n}\right\|^{2} \leq & \left(1-\beta_{n} \bar{\gamma}\right)^{2}\left\|z_{n}-p\right\|^{2}-\left\|y_{n}-p\right\|^{2} \\
& +2 \gamma_{n}\left\|z_{n}-t_{n}\right\|\left\|B z_{n}-B p\right\|-\gamma_{n}^{2}\left\|B z_{n}-B p\right\|^{2}+c_{n} \\
\leq & \left(1-\beta_{n} \bar{\gamma}\right)^{2}\left\|u_{n}-p\right\|^{2}-\left\|y_{n}-p\right\|^{2}+2 \gamma_{n}\left\|z_{n}-t_{n}\right\|\left\|B z_{n}-B p\right\| \\
& -\gamma_{n}^{2}\left\|B z_{n}-B p\right\|^{2}+c_{n} \\
\leq & \left(1-\beta_{n} \bar{\gamma}\right)^{2}\left[\left\|u_{n}-y_{n}\right\|^{2}+2\left\|y_{n}-u_{n}\right\|\left\|y_{n}-p\right\|+\left\|y_{n}-p\right\|^{2}\right]-\left\|y_{n}-p\right\|^{2} \\
& +2 \gamma_{n}\left\|z_{n}-t_{n}\right\|\left\|B z_{n}-B p\right\|-\gamma_{n}^{2}\left\|B z_{n}-B p\right\|^{2}+c_{n},
\end{aligned}
$$

so, $\lim _{n \rightarrow \infty}\left\|z_{n}-t_{n}\right\|=0$.

Also,

$$
\begin{aligned}
\left\|W_{n} y_{n}-y_{n}\right\| \leq & \left\|y_{n}-z_{n}\right\|+\left\|z_{n}-W_{n} y_{n}\right\| \\
\leq & \left\|y_{n}-z_{n}\right\|+\left\|z_{n}-W_{n} u_{n}\right\|+\left\|W_{n} u_{n}-W_{n} y_{n}\right\| \\
\leq & \left\|y_{n}-z_{n}\right\|+\lambda_{n}\left\|u_{n}-W_{n} u_{n}\right\|+\left\|u_{n}-y_{n}\right\| \\
\leq & \left\|y_{n}-z_{n}\right\|+\lambda_{n}\left\|u_{n}-y_{n}\right\|+\lambda_{n}\left\|y_{n}-W_{n} u_{n}\right\|+\left\|u_{n}-y_{n}\right\| \\
\leq & \left\|y_{n}-z_{n}\right\|+\left(1+\lambda_{n}\right)\left\|u_{n}-y_{n}\right\|+\lambda_{n}\left\|y_{n}-W_{n} y_{n}\right\| \\
& +\lambda_{n}\left\|W_{n} y_{n}-W_{n} u_{n}\right\| .
\end{aligned}
$$

So,

$\left(1-\lambda_{n}\right)\left\|W_{n} y_{n}-y_{n}\right\| \leq\left\|y_{n}-z_{n}\right\|+\left(2+\lambda_{n}\right)\left\|u_{n}-y_{n}\right\|$, by (2.14) and (2.11) we have,

$$
\left\|W_{n} y_{n}-y_{n}\right\| \rightarrow 0
$$

By lemma (1.8) and (2.15), we obtain,

$$
\begin{aligned}
\left\|W y_{n}-y_{n}\right\| & \leq\left\|W y_{n}-W_{n} y_{n}\right\|+\left\|W_{n} y_{n}-y_{n}\right\| \\
& \leq \sup _{y \in\left\{y_{n}\right\}}\left\|W y-W_{n} y\right\|+\left\|W_{n} y_{n}-y_{n}\right\| \rightarrow 0, \text { as } n \rightarrow \infty .
\end{aligned}
$$

Finally we have,

$\left\|W t_{n}-t_{n}\right\| \leq\left\|W t_{n}-W y_{n}\right\|+\left\|W y_{n}-y_{n}\right\|+\left\|y_{n}-t_{n}\right\|$. So, $\left\|W t_{n}-t_{n}\right\| \rightarrow 0$.

Observe that, $P_{F}(\gamma f+(I-A))$ is a contraction, in fact for $x, y \in H$ we have,

$$
\begin{aligned}
\left\|P_{F}(\gamma f+(I-A))(x)-P_{F}(\gamma f+(I-A))(y)\right\| & \leq\|(\gamma f+(I-A))(x)-(\gamma f+(I-A))(y)\| \\
& \leq \gamma\|f(x)-f(y)\|+\|I-A\|\|x-y\| \\
& \leq \gamma \alpha\|x-y\|+(1-\bar{\gamma})\|x-y\| \\
& =[1-(\bar{\gamma}-\gamma \alpha)]\|x-y\| .
\end{aligned}
$$

Banach's Contraction Mapping Principle guarantees that $P_{F}(\gamma f+(I-A))$ has a unique fixed point, say $q \in H$. That is, $P_{F}(\gamma f+(I-A))(q)=q$. 
Now we choose a subsequence $\left\{t_{n_{i}}\right\}$ of $\left\{t_{n}\right\}$ such that,

$$
\limsup _{n \rightarrow \infty}\left\langle\gamma f(q)-A q, W t_{n}-q\right\rangle=\lim _{i \rightarrow \infty}\left\langle\gamma f(q)-A q, W t_{n_{i}}-q\right\rangle .
$$

Since $\left\{t_{n_{i}}\right\}$ is bounded, there exists a subsequence $\left\{t_{n_{i_{j}}}\right\}$ of $\left\{t_{n_{i}}\right\}$ which converges weakly to $z \in C$. Without loss of generality, we can assume that $t_{n_{i}} \rightarrow z$. From $\left\|W t_{n_{i}}-t_{n_{i}}\right\| \rightarrow 0$, we obtain $W t_{n_{i}} \rightarrow z$. Therefore, we have

$$
\begin{aligned}
\limsup _{n \rightarrow \infty}\left\langle\gamma f(q)-A q, W t_{n}-q\right\rangle & =\lim _{i \rightarrow \infty}\left\langle\gamma f(q)-A q, W t_{n_{i}}-q\right\rangle \\
& =\langle\gamma f(q)-A q, z-q\rangle .
\end{aligned}
$$

We want to prove that $z \in F(W)=\bigcap_{i=1}^{\infty} F\left(T_{i}\right)$. Suppose $z \notin F(W)$, that is $W z \neq z$. Since $t_{n_{i}} \rightarrow z$, by Opial's condition and (2.16) we have,

$$
\begin{aligned}
\liminf _{i \rightarrow \infty}\left\|t_{n_{i}}-z\right\| & <\liminf _{i \rightarrow \infty}\left\|t_{n_{i}}-W z\right\| \\
& \leq \liminf _{i \rightarrow \infty}\left\{\left\|t_{n_{i}}-W t_{n_{i}}\right\|+\left\|W t_{n_{i}}-W z\right\|\right\} \\
& \leq \liminf _{i \rightarrow \infty}\left\{\left\|t_{n_{i}}-W t_{n_{i}}\right\|+\left\|t_{n_{i}}-z\right\|\right\} \\
& =\liminf _{i \rightarrow \infty}\left\|t_{n_{i}}-z\right\| .
\end{aligned}
$$

That is a contradiction, hence $z \in F(W)=\bigcap_{i=1}^{\infty} F\left(T_{i}\right)$.

Next we prove that $z \in E P(F)$. Since $u_{n}=T_{r_{n}}$, we have,

$$
F\left(u_{n}, y\right)+\frac{1}{r_{n}}\left\langle y-u_{n}, u_{n}-x_{n}\right\rangle \geq 0, \forall y \in C .
$$

From (A2), we have,

$$
\frac{1}{r_{n}}\left\langle y-u_{n}, u_{n}-x_{n}\right\rangle \geq F\left(y, u_{n}\right)
$$

and hence,

$$
\left\langle y-u_{n_{i}}, \frac{u_{n_{i}}-x_{n_{i}}}{r_{n_{i}}}\right\rangle \geq F\left(y, u_{n_{i}}\right) .
$$

From $\left\|u_{n}-x_{n}\right\| \rightarrow 0,\left\|x_{n}-W t_{n}\right\| \rightarrow 0$ and $\left\|W t_{n}-t_{n}\right\| \rightarrow 0$ we get $u_{n_{i}} \rightarrow z$.Since $\frac{u_{n_{i}}-x_{n_{i}}}{r_{n_{i}}} \rightarrow 0$ by (A4) we have, $F(y, z) \leq 0, \forall y \in C$.

For $t$ with $0<t \leq 1$ and $y \in C$, let $y_{t}=t y+(1-t) z$. Since $y \in C$ and $z \in C$ we have $y_{t} \in C$ and hence $F\left(y_{t}, z\right) \leq 0$. So from (A1) and (A4) we have

$$
0=F\left(y_{t}, y_{t}\right) \leq t F\left(y_{t}, y\right)+(1-t) F\left(y_{t}, z\right) \leq t F\left(y_{t}, y\right)
$$

and hence $0 \leq F\left(y_{t}, y\right)$. From (A3) we have $0 \leq F(z, y)$ for all $y \in C$ so, $z \in E P(F)$.

Finally, similar as the proof of [9, Theorem 2.1, pp. 678], we can prove that $z \in V I(B, C)$. Therefore

$$
z \in F(W) \cap V I(B, C) \cap E P(F) .
$$


Moreover by (2.17), we have,

$$
\limsup _{n \rightarrow \infty}\left\langle\gamma f(q)-A q, W t_{n}-q\right\rangle=\langle\gamma f(q)-A q, z-q\rangle \leq 0 .
$$

Also,

$$
\begin{aligned}
\limsup _{n \rightarrow \infty}\left\langle\gamma f(q)-A q, t_{n}-q\right\rangle & =\limsup _{n \rightarrow \infty}\left\langle\gamma f(q)-A q,\left(t_{n}-W t_{n}\right)+\left(W t_{n}-q\right)\right\rangle \\
& \leq \limsup _{n \rightarrow \infty}\left\langle\gamma f(q)-A q, W t_{n}-q\right\rangle \\
& \leq 0
\end{aligned}
$$

Hence we have,

$$
\begin{aligned}
\limsup _{n \rightarrow \infty}\left\langle\gamma f(q)-A q, x_{n}-q\right\rangle= & \left\langle\gamma f(q)-A q, x_{n}-y_{n}\right\rangle \\
& +\left\langle\gamma f(q)-A q, y_{n}-t_{n}\right\rangle+\left\langle\gamma f(q)-A q, t_{n}-q\right\rangle,
\end{aligned}
$$

by (2.13) and (2.18) we have, $\lim \sup _{n \rightarrow \infty}\left\langle\gamma f(q)-A q, x_{n}-q\right\rangle \leq 0$.

On the other hand we have, $\left\langle\gamma f(q)-A q, y_{n}-q\right\rangle=\left\langle\gamma f(q)-A q, y_{n}-x_{n}\right\rangle+\left\langle\gamma f(q)-A q, x_{n}-q\right\rangle$, by (2.13) and (2.19) we have,

$$
\limsup _{n \rightarrow \infty}\left\langle\gamma f(q)-A q, y_{n}-q\right\rangle \leq 0
$$

So,

$$
\begin{aligned}
\left\|y_{n}-q\right\|^{2}= & \left\|\beta_{n} \gamma f\left(z_{n}\right)+\left(I-\beta_{n} A\right) t_{n}-q\right\|^{2} \\
= & \left\|\left(I-\beta_{n} A\right)\left(t_{n}-q\right)+\beta_{n}\left(\gamma f\left(z_{n}\right)-A q\right)\right\|^{2} \\
\leq & \left\|\left(I-\beta_{n} A\right)\left(t_{n}-q\right)\right\|^{2}+2 \beta_{n}\left\langle\gamma f\left(z_{n}\right)-A q, y_{n}-q\right\rangle \\
= & \left\|\left(I-\beta_{n} A\right)\left(t_{n}-q\right)\right\|^{2}+2 \beta_{n}\left\langle\gamma f\left(z_{n}\right)-\gamma f(q), y_{n}-q\right\rangle \\
& +2 \beta_{n}\left\langle\gamma f(q)-A q, y_{n}-q\right\rangle \\
\leq & \left(1-\beta_{n} \bar{\gamma}\right)^{2}\left\|t_{n}-q\right\|^{2}+2 \beta_{n} \gamma \alpha\left\|z_{n}-q\right\|\left\|y_{n}-q\right\| \\
& +2 \beta_{n}\left\langle\gamma f(q)-A q, y_{n}-q\right\rangle \\
\leq & \left(1-\beta_{n} \bar{\gamma}\right)^{2}\left\|x_{n}-q\right\|^{2}+2 \beta_{n} \gamma \alpha\left\|x_{n}-q\right\|\left\|y_{n}-q\right\| \\
& +2 \beta_{n}\left\langle\gamma f(q)-A q, y_{n}-q\right\rangle \\
\leq & \left(1-\beta_{n} \bar{\gamma}\right)^{2}\left\|x_{n}-q\right\|^{2}+\beta_{n} \gamma \alpha\left(\left\|x_{n}-q\right\|^{2}+\left\|y_{n}-q\right\|^{2}\right) \\
& +2 \beta_{n}\left\langle\gamma f(q)-A q, y_{n}-q\right\rangle,
\end{aligned}
$$

so, 


$$
\begin{aligned}
\left\|y_{n}-q\right\|^{2} \leq & \frac{\left(1-\beta_{n} \bar{\gamma}\right)^{2}+\beta_{n} \gamma \alpha}{1-\beta_{n} \gamma \alpha}\left\|x_{n}-q\right\|^{2} \\
& +\frac{2 \beta_{n}}{1-\beta_{n} \gamma \alpha}\left\langle\gamma f(q)-A q, y_{n}-q\right\rangle \\
\leq & {\left[1-\frac{2 \beta_{n}(\bar{\gamma}-\gamma \alpha)}{1-\beta_{n} \gamma \alpha}\right]\left\|x_{n}-q\right\|^{2} } \\
& +\frac{2 \beta_{n}(\bar{\gamma}-\gamma \alpha)}{1-\beta_{n} \gamma \alpha}\left[\frac{1}{\bar{\gamma}-\gamma \alpha}\left\langle\gamma f(q)-A q, y_{n}-q\right\rangle+\frac{\beta_{n} \bar{\gamma}^{2}}{2(\bar{\gamma}-\gamma \alpha)} M_{4}\right],
\end{aligned}
$$

where $M_{4}$ is an appropriate constant such that $M_{4} \geq \sup _{n \geq 1}\left\{\left\|x_{n}-q\right\|\right\}$.

Finally,

$$
\begin{aligned}
\left\|x_{n+1}-q\right\|^{2} \leq & \alpha_{n}\left\|x_{n}-q\right\|^{2}+\left(1-\alpha_{n}\right)\left\|y_{n}-q\right\|^{2} \\
\leq & {\left[1-\left(1-\alpha_{n}\right) \frac{2 \beta_{n}(\bar{\gamma}-\gamma \alpha)}{1-\beta_{n} \gamma \alpha}\left\|x_{n}-q\right\|^{2}\right.} \\
& +\left(1-\alpha_{n}\right) \frac{2 \beta_{n}(\bar{\gamma}-\gamma \alpha)}{1-\beta_{n} \gamma \alpha}\left[\frac{1}{\bar{\gamma}-\gamma \alpha}\left\langle\gamma f(q)-A q, y_{n}-q\right\rangle+\frac{\beta_{n} \bar{\gamma}}{2(\bar{\gamma}-\gamma \alpha)} M_{4}\right],
\end{aligned}
$$

we put $h_{n}=\left(1-\alpha_{n}\right) \frac{2 \beta_{n}(\bar{\gamma}-\gamma \alpha)}{1-\beta_{n} \gamma \alpha}$ and $r_{n}=\frac{1}{\bar{\gamma}-\gamma \alpha}\left\langle\gamma f(q)-A q, y_{n}-q\right\rangle+\frac{\beta_{n} \bar{\gamma}}{2(\bar{\gamma}-\gamma \alpha)} M_{4}$,

so,

$$
\left\|x_{n+1}-q\right\|^{2} \leq\left(1-h_{n}\right)\left\|x_{n}-q\right\|^{2}+t_{n}
$$

where $t_{n}=h_{n} r_{n}$, from condition (C1) we have $\lim _{n \rightarrow \infty} h_{n}=0$ and by(2.20), $\limsup _{n \rightarrow \infty} t_{n} \leq 0$.

Finally lemma (1.2), implies $x_{n} \rightarrow 0$ as $n \rightarrow \infty$.

If we put $T_{n} x=x$ for all $n=1,2, \ldots, k$ and for all $x \in C$ in (1.4) we have $W_{n} x=x$, then we have,

Corollary 2.1. Let $H$ be a real Hilbert space. Let $F$ be a bifunction from $C \times C \longrightarrow \mathbb{R}$ satisfying (A1)(A4) and let $B$ be an $\alpha$-inverse-strongly monotone mapping of $C$ into $H, F:=V I(B, C) \cap E P(F) \neq \emptyset$. Suppose $A$ is a strongly positive linear bounded self-adjoint operator with the coefficient $\bar{\gamma} \geq 0$ and $0<\gamma \leq \overline{\bar{\gamma}}$. Let $\left\{\alpha_{n}\right\},\left\{\beta_{n}\right\},\left\{\lambda_{n}\right\}$ and $\left\{\delta_{n}\right\}$ be sequences in $[0,1]$ satisfying the following conditions:

(C1) $\sum_{n=0}^{\infty} \beta_{n}=\infty, \lim _{n \rightarrow \infty} \beta_{n}=0$;

(C2) $\sum_{i=1}^{\infty}\left|\lambda_{n}-\lambda_{n+1}\right|<\infty$;

(C3) $\sum_{i=1}^{\infty}\left|\alpha_{n}-\alpha_{n+1}\right|<\infty$;

(C4) $\exists \lambda \in[0,1] ; \lambda_{n}<\lambda, \forall n \geq 1$;

(C5) $\sum_{n=1}^{\infty}\left|\gamma_{n}-\gamma_{n+1}\right|<\infty, \gamma_{n} \in[a, b], a, b \in(0,2 \alpha)$;

(C6) $\liminf _{n \rightarrow \infty} r_{n}>0$ and $\sum_{i=1}^{\infty}\left|r_{n+1}-r_{n}\right|<\infty$.

Then the sequences $\left\{x_{n}\right\}$ defined by

$$
\begin{aligned}
& F\left(u_{n}, y\right)+\frac{1}{r_{n}}\left\langle y-u_{n}, u_{n}-x_{n}\right\rangle \geq 0, \forall y \in C, \\
& y_{n}=\beta_{n} \gamma f\left(u_{n}\right)+\left(I-\beta_{n} A\right) P_{C}\left(u_{n}-\gamma_{n} B u_{n}\right), \\
& x_{n+1}=\alpha_{n} x_{n}+\left(1-\alpha_{n}\right) y_{n},
\end{aligned}
$$

converges strongly to $q \in F$, where $q=P_{F}(\gamma f+(I-A))(q)$ which solves the following variational inequality:

$$
\langle\gamma f(q)-A q, p-q\rangle \leq 0, \forall p \in F .
$$


If we put $F(x, y)=0$ for all $x, y \in C$ and $r_{n}=1$ for all $n \in \mathbb{N}$ in theorem (2.1), then we have,

Corollary 2.2. Let $H$ be a real Hilbert space. Let $\left\{T_{i}: C \longrightarrow C\right\}$ be a infinite family of nonexpansive mappings with $F:=\bigcap_{i=1}^{\infty} F\left(T_{i}\right) \bigcap V I(B, C) \neq \emptyset$. Suppose $A$ is a strongly positive linear bounded selfadjoint operator with the coefficient $\bar{\gamma} \geq 0$ and $0<\gamma \leq \frac{\bar{\gamma}}{\alpha}$. Let $\left\{\alpha_{n}\right\},\left\{\beta_{n}\right\},\left\{\lambda_{n}\right\}$ and $\left\{\delta_{n}\right\}$ be sequences in $[0,1]$ satisfying the following conditions:

(C1) $\sum_{n=0}^{\infty} \beta_{n}=\infty, \lim _{n \rightarrow \infty} \beta_{n}=0$;

(C2) $\sum_{i=1}^{\infty}\left|\lambda_{n}-\lambda_{n+1}\right|<\infty$;

(C3) $\sum_{i=1}^{\infty}\left|\alpha_{n}-\alpha_{n+1}\right|<\infty$;

(C4) $\exists \lambda \in[0,1] ; \lambda_{n}<\lambda, \forall n \geq 1$;

(C5) $\sum_{n=1}^{\infty}\left|\gamma_{n}-\gamma_{n+1}\right|<\infty, \gamma_{n} \in[a, b], a, b \in(0,2 \alpha)$;

Then the sequences $\left\{x_{n}\right\}$ defined by

$$
\begin{aligned}
& z_{n}=\lambda_{n} u_{n}+\left(1-\lambda_{n}\right) W_{n} u_{n}, \\
& y_{n}=\beta_{n} \gamma f\left(z_{n}\right)+\left(I-\beta_{n} A\right) P_{C}\left(z_{n}-\gamma_{n} B z_{n}\right), \\
& x_{n+1}=\alpha_{n} x_{n}+\left(1-\alpha_{n}\right) y_{n},
\end{aligned}
$$

converges strongly to $q \in F$, where $q=P_{F}(\gamma f+(I-A))(q)$ which solves the following variational inequality:

$$
\langle\gamma f(q)-A q, p-q\rangle \leq 0, \forall p \in F
$$

\section{References}

[1] E. Blum, W. Oettli, From optimization and variational inequalities to equilibrium problems, Math. Student 63 (1994) 123-145.

[2] P.L. Combettes, S.A. Hirstoaga, Equilibrium programming using proximal-like algorithms, Math. Program. 79 (1997) 29-41.

[3] G.Marino, H. K. Xu, A general iterative method for nonexpansive mappings in Hilbert spaces, J. Math. Anal. appl, 318 (2006) 43-52.

[4] S. Plubtieng, R. Punpaeng, A general iterative method for equilibrium problems and fixed point problems in Hilbert spaces, J. Math. Anal. Appl. 336 (2007) 455469.

[5] X. Qin, Y. Cho, S. Kang, An iterative method for an infinite family of nonexpansive mappings in Hilbert spaces, Bull. Malays. Math. Sci. Soc. 32 (2) (2009) 161171.

[6] K. Shimoji, W.Takahashi, Strong convergence to common fixed points of infinite nonexpansive mappings and applications, Taiwanese J. Math, 5 (2) (2001) 387-404.

[7] T. Suzuki, Strong convergence of Krasnoselskii and Manns type sequences for one-parameter nonexpansive semigroups without Bochner integrals, J. Math. Anal. Appl. 305 (1) (2005) 227-239.

[8] S. Takahashi, W. Takahashi, Viscosity approximation methods for equilibrium problems and fixed point problems in Hilbert spaces, J. Math. Anal. Appl. 331 (2007) 506515.

[9] R. Wangkeeree, U.Kamraska, A general iterative method for solving the variational inequality problem and fixed point problem of an infinite family of nonexpansive mappings in Hilbert spaces, Fixed Point Theory and Applications. Article ID 369215, (2009) 23 pages.

[10] H. K. Xu, An iterative approach to quadratic optimization, Journal of Optimization Theory and Applications, 116 (3) (2003) 569-678. 
[11] J. Zhao, S. He, A new iterative method for equilibrium problems and fixed point problems of infinitely nonexpansive mappings and monotone mappings, Journal of Applied Mathematics and Computation, 215 (2009) 670-680. 